\title{
Power Switching Protocol for Two-way Relaying Network under Hardware Impairments
}

\author{
Dinh-Thuan DO \\ Duy Tan University, Da Nang City, Vietnam \\ dodinhthuan@gmail.com
}

\begin{abstract}
In this paper, we analyze the impact of hardware impairments at relay node and source node (i.e. imperfect nodes) on network performance by evaluating outage probability based on the effective signal to noise and distortion ratio (SNDR). Especially, we propose energy harvesting protocol at the relay and source nodes, namely, power switching imperfect relay (PSIR) and power switching imperfect source (PSIS). Aiming to determine the performance of energy constrained network, we first derive closed-form expressions of the outage probability and then the throughput can be maximized in delay-limited transmission mode. The simulation results provide practical insights into the impacts of hardware impairments and power switching factors of the energy harvesting protocol on the performance of energy harvesting enabled two-way relaying network.
\end{abstract}

\section{Keywords}

Amplify-and-forward, energy harvesting, two-way relaying network, wireless powered communication networks

\section{Introduction}

Recently, energy harvesting devices have received extensive attentions to green communications thanks to significant advantages over traditional grid-powered and battery-based devices [1]. Utilizing the wireless power transfer technique can avoid implementing cables which are difficulties in the conventional networks. Alternatively, mobile nodes can harvest energy from ambient sources such as radio frequency (RF), solar, wind, motion effects, thermoelectric effects or other physical phenomena. Besides common renewable energy sources such as sun and wind, the ambient RF signal is utilized at the receiver antennas and can be applied in wireless power transfer. Therefore, RF signal is introduced as appealing vehicle for simultaneous information and power transfer. In such green communications, the rectifying antenna receives RF signal and then transfers to rectifying circuit to produce direct current (DC) voltage. However, path loss and lower efficiency of RF-DC conversion are the main problem leading to low wireless energy transfer efficiency. The alternative RF-based energy resource provides proper solutions in the field of low-power equipments of wireless sensor networks (WSN). It is often difficult to replace the limited lifetime batteries in large number of sensor nodes in order to maintain the long-term operation of WSN. Therefore, energy harvesting techniques are emerging solution to overcome the crucial battery problem.

A new research trend on wireless power transfer is combined by the energy harvesting technology and the conventional cooperative networks subject to prolong the lifetime and expand the coverage [2]. Regarding cellular networks and WLAN, the batteries in handheld devices have limited operation time, meanwhile the fast growth of multimedia applications consumes too much energy. Energy harvesting techniques play an important role in wireless powered communication networks, in which the relay nodes are assumed not to have internal energy source.

In the context of bidirectional transmission in cooperative communications so-called two-way relaying networks (TWRN) are used where two source nodes simultaneously transfer information and energy to the relay node. To apply energy harvesting to two-way relay transmission, the transmission process is separated into two phases. During the first phase, namely Multiple Access (MA) phase, the two source nodes transmit information and energy to the relay node. Afterward, in the broadcast (BC) phase, the relay node transmits information simultaneously to both the source nodes. Recently, in [3], the authors shown that joint power optimization problem and relay selection can be solved for the maximization of the throughput of an amplify-and-forward (AF) relaying network with energy harvested at the relay node. Regarding cognitive radio system, it was shown in [4] that the optimal cooperation scheme can maximize the throughput of secondary user (SU) which harvests energy from RF signal. The other important parameter investigated in [5] is the rate-energy trade-off factor between the maximum ergodic capacity and maximum harvested energy of SU who shares the spectrum and harvests energy from the primary transmission. Exploring the other special ambient energy resource, the authors in [6] developed cooperative network with interference aided energy harvesting relay which harvests energy from the co-channel interference (CCI) signal. The authors in [6] also derived the analytical ex- 
pressions for ergodic capacity based on the probability density function of the random variables in terms of signal noise ratio (SNR) on each hop for performance evaluation. In addition, the optimized time splitting coefficient analyzed in [7] can substantially boost the throughput of the full-duplex cooperative networks in both cases of AF and decode-and-forward (DF) protocols. To analyze energy policies, it was shown in [8] the performance of optimal throughput in one-way relaying network was impacted by time switching and power splitting coefficients in the time power switching protocol (TPSR). In the other protocols of relaying network, the authors in [9] derived expressions of average achieved sum-rates for DF and compute-and-forward in order to reflect an energy constraint. It is also worth pointing out that in [10] the authors proposed two energy constraint relaying protocols, namely, i) time switching-based relaying (TSR) protocol and ii) power splitting-based relaying protocol (PSR) in wireless energy harvesting and information processing network. Related to design structure of energy harvesting circuits [11], [12], the authors analyzed the energy performance in terms of integrated circuit power consumption.

However, the vast majority of previous works in [3], [13-17] assumed that the transceiver hardware is ideal for evaluating the performance of relaying network. In practice, the most mobile equipments suffer from hardware impairments, e.g. phase noise, in-phase and quadraturephase imbalance (IQI), nonlinear power amplifier [18]. The analysis of hardware impairments in energy harvesting enabled cooperative communication has not been addressed in the literature. Hardware impairments are particularly prone by the cheap equipments. It is worth noting that hardware impairments limit the system's performance such as the capacity, throughput and symbol error rate (SER). In addition, the relaying network is not only affected by non-ideal hardware but also by channel estimation accuracy and signal processing complexity [14], [19].

In this paper, we deploy two-way AF relay as lowcomplexity protocol that amplifies the received signal without decoding processing. We formulate and examine the outage probability and the maximized throughput for the proposed power switching imperfect relay (PSIR) and power switching imperfect source (PSIS) protocols with delay-limited transmission mode. Motivated by the results in [20], we hereafter propose a general expression of outage probability under hardware impairments for all proposed energy harvesting schemes. Our mathematical and simulation analyses provide how the performance of communications changes under the different levels of hardware impairments and energy harvesting efficiency factors.

The main contributions of this paper are summarized as follows:

- Based on the conventional energy harvesting structure (i.e. power switching based relay), we propose two main energy harvesting protocols (PSIR, PSIS) which show the principles of collecting power, especially in low cost receiver with high level of hardware impairments.

- For the PSIR and PSIS protocols, we analyze how unwanted noise of non-ideal hardware leads to degrade the effective SNDR (signal to noise and distortion ratio) and hence harming the outage probability. Interestingly, we obtain the tractable closed-form of outage probability in general expression for all proposed protocols.

- We derive analytical formula for the maximized throughput by changing energy harvesting fractions in calculation of outage probability under delay-limited transmission mode. More importantly, the derived expressions lead to obtain the optimal value of power switching factors in the proposed protocols.

The remainder of this paper is organized as follows: Section 2 introduces the system model and illustrates the energy harvesting scheme for the relay in TWRN. In Sec. 3, we examine the closed-form expression of SNDR at the two source nodes of energy harvesting relaying network under the impact of hardware impairments. The tractable formula for outage probability and optimal throughput are derived in Sec. 4. The analysis and simulation results are dedicated to the performance of imperfect source and relay affected by non-ideal hardware. Our numerical results to corroborate the theoretical expressions are illustrated in Sec. 5. Finally, the main discussion is summarized in Sec. 6.

\section{Signal and System Model}

This paper considers TWRN with all nodes equipped with single antenna [15], assuming that only the relay node has no embedded power supply. They use harvested energy in the first phase in order to transmit information in the second phase. The TWRN is analyzed based on AF protocol with two hops including two transmitter nodes ( $A$ and $B$ ) and a relay node $(R)$. After the energy constrained relay node harvests power from the RF signal transmitted by both of source nodes (i.e., the transmitter nodes), it utilizes the harvested energy in order to amplify and forward the received signal from the relay to the destination nodes. Therefore, the process of communications is performed in two time slots in which the first slot is assigned to the source-relay link and the second slot is designed to the relay-destination link.

For the ease of implementation, the assumptions for the TWRN system model and the proposed schemes are given as follows:

- (A1.) The destination node is only received directly by relay node without any signal from the source node. In addition, the AF relaying scheme is chosen at the intermediate relay.

- (A2.) The channel state information is estimated per- 
fectly at the relay and destination nodes. We assume only small number of pilots for training sequences and remaining bandwidth at acceptable limitation for transmitting pure data.

- (A3.) It can be measured with some kinds of equipments in term of level of hardware impairments $\tau_{A}$ and $\tau_{R}$ at the source and relay nodes, respectively.

- (A4.) In the TWRN, we also focus on multiple access broadcast (MABC) protocol which is more spectral efficient than time division broadcast (TDBC) protocol. In MABC, the signal is transmitted from both the source nodes to the relay node in the first half period and then broadcast signal is conducted from relay to both the destination nodes in the second half period.

- (A5.) All channels in the TWRN are considered as slow and frequency flat fading. In addition, these independent Rayleigh fading channel coefficients are assumed to be constant over the block time $T$ of signal frame.

Conventionally in wireless systems, the received signals at the relay and destination nodes are modeled as

$$
y_{i}=h_{i} x_{i}+v_{i}+n_{i}, \quad i=A, B, R,
$$

where $h_{i}$ is the channel which is often estimated by training sequences prior to sending useful data and $n_{i}$ is the Gaussian noise, while $v_{i}$ is defined as distortion noise from impairments in the transceivers. In this paper, we consider the effect of hardware impairments on both the source and relay nodes (i.e. imperfect source, imperfect relay). We denote $h_{A}$ and $h_{B}$ as the channel coefficients from the node $A$ to the node $R$ and the node $B$ to the node $R$, respectively and $\Omega_{j}=\mathbb{E}_{h_{j}}\left\{\left|h_{j}\right|^{2}\right\}, j=A, B$ is the average gain, or we often represent $h_{j} \sim C N\left(0, \Omega_{j}\right)$. The system works under Rayleigh fading channel and average gains have been modeled with respect to the probability density function (PDF) and cumulative density function (CDF) respectively as follows

$$
\begin{gathered}
f_{\left|h_{j}\right|^{2}}(x)=\frac{1}{\Omega_{i}} e^{-\frac{x}{\Omega_{j}}}, \quad x \geq 0, \\
F_{\left|h_{j}\right|^{2}}(x)=1-e^{-\frac{x}{\Omega_{j}}}, \quad x \geq 0 .
\end{gathered}
$$

\section{Power Switching Protocol}

\subsection{Power Switching Imperfect Relay Protocol}

In PSIR protocol, the information transmission from the source node to the relay node occurs in the first block time $T / 2$ and the remaining one of $T / 2$ is used for informa- tion processing between the relay and the destination nodes. In addition, the fraction of the received power used for energy harvesting is denoted by $\beta P_{j}(j=A, B)$ with $0 \leq \beta \leq 1$ and the remaining power of $(1-\beta) P_{j}$ is supplied for transferring information from the source node to the relay node. The harvested energy at the power constraint relay can be rewritten as

$$
E_{h}=\eta \beta\left(P_{A}\left|h_{A}\right|^{2}+P_{B}\left|h_{B}\right|^{2}\right) T / 2
$$

where $\eta$ denotes energy conversion efficiency. Indeed, the harvested energy which is then transmitted in duration of $T / 2$ from the relay node can be calculated as

$$
P_{R}=\frac{E_{h}}{T / 2}=\eta \beta\left(P_{A}\left|h_{A}\right|^{2}+P_{B}\left|h_{B}\right|^{2}\right) .
$$

In the PSIR, the power for transmitting information by the source nodes is only allocated a fraction of $(1-\beta) P_{j}$. Thus, the received signal at the energy constraint relay can be expressed as follows

$$
y_{R}=\sqrt{(1-\beta) P_{A}} h_{A} x_{A}+\sqrt{(1-\beta) P_{B}} h_{B} x_{B}+v_{R}+n_{R} .
$$

For simplicity, it is assumed that all nodes have equal noise variances denoted as $\sigma^{2}$. In the TWRN with hardware impairment constraint at the imperfect relay node, it amplifies the received signal with the modified variable gain given by

$$
G^{-1}=\sqrt{\left(P_{A}\left|h_{A}\right|^{2}+P_{B}\left|h_{B}\right|^{2}\right)\left(1+\tau_{R}^{2}\right)+\sigma^{2}} .
$$

The received signals at the two destination nodes are given by

$$
\begin{aligned}
& y_{A}=\sqrt{P_{R}} h_{A} G \times \\
& \left(\sqrt{(1-\beta) P_{A}} h_{A} x_{A}+\sqrt{(1-\beta) P_{B}} h_{B} x_{B}+v_{R}+n_{R}\right)+n_{A} \\
& =\sqrt{P_{R}} \sqrt{(1-\beta) P_{A}} G\left|h_{A}\right|^{2} x_{A}+\sqrt{P_{R}} \sqrt{(1-\beta) P_{B}} h_{A} h_{B} G x_{B} \\
& +\sqrt{P_{R}} G h_{A} v_{R}+\sqrt{P_{R}} G h_{A} n_{R}+n_{A},
\end{aligned}
$$

and

$$
\begin{aligned}
& y_{B}=\sqrt{P_{R}} h_{B} G \times \\
& \left(\sqrt{(1-\beta) P_{A}} h_{A} x_{A}+\sqrt{(1-\beta) P_{B}} h_{B} x_{B}+v_{R}+n_{R}\right)+n_{B} \\
& =\sqrt{P_{R}} \sqrt{(1-\beta) P_{B}} G\left|h_{B}\right|^{2} x_{B}+\sqrt{P_{R}} \sqrt{(1-\beta) P_{A}} h_{A} h_{B} G x_{A} \\
& +\sqrt{P_{R}} G h_{B} v_{R}+\sqrt{P_{R}} G h_{B} n_{R}+n_{B} .
\end{aligned}
$$

It is worth noting that the terms $\sqrt{P_{R}} \sqrt{(1-\beta) P_{A}} G\left|h_{A}\right|^{2} x_{A}$ and $\sqrt{P_{R}} \sqrt{(1-\beta) P_{B}} G\left|h_{B}\right|^{2} x_{B}$ in (8) and (9) respectively can be suppressed by applying network coding technique [21]. Thus, the end-to-end SNDR at source node $A$ can be expressed as

$$
\gamma_{A 1}=\frac{(1-\beta) P_{B}\left|h_{A}\right|^{2}\left|h_{B}\right|^{2}}{\left|h_{A}\right|^{2}\left(P_{A}\left|h_{A}\right|^{2}+P_{B}\left|h_{B}\right|^{2}\right) \tau_{R}^{2}+\left|h_{A}\right|^{2} \sigma^{2}+N_{1}+N_{2}}
$$


where $N_{1}=\frac{\sigma^{2}\left(1+\tau_{R}^{2}\right)}{\eta \beta}, N_{2}=\frac{\sigma^{4}}{\eta \beta\left(P_{A}\left|h_{A}\right|^{2}+P_{B}\left|h_{B}\right|^{2}\right)}$.

Next, we consider the TWRN at high SNR and hence the term $\mathrm{N}_{2}$ is negligible to reduce the complexity in calculation. Consequently, the closed-form expression of SNDR can be derived as follows

$$
\gamma_{A 1}^{*}=\frac{\left|h_{A}\right|^{2}\left|h_{B}\right|^{2}}{\frac{\tau_{R}^{2} P_{A}\left|h_{A}\right|^{4}}{(1-\beta) P_{B}}+\frac{\tau_{R}^{2}\left|h_{A}\right|^{2}\left|h_{B}\right|^{2}}{1-\beta}+\frac{\sigma^{2}\left|h_{A}\right|^{2}}{(1-\beta) P_{B}}+\frac{N_{1}}{P_{B}(1-\beta)}} .
$$

\subsection{Power Switching Imperfect Source Protocol}

In the PSIS protocol, the received signal at the energy constraint relay node can be expressed as follows

$$
y_{R}=\sqrt{(1-\beta) P_{A}} h_{A} x_{A}+\sqrt{(1-\beta) P_{B}} h_{B} x_{B}+n_{R}
$$

The received signals at the two source nodes in this case are formulated as

$$
\begin{aligned}
& y_{A}=\sqrt{P_{R}} h_{A} G\left(\sqrt{(1-\beta) P_{A}} h_{A} x_{A}+\sqrt{(1-\beta) P_{B}} h_{B} x_{B}+n_{R}\right)+ \\
& +v_{A}+n_{A} \\
& =\sqrt{P_{R}} \sqrt{(1-\beta) P_{A}} G\left|h_{A}\right|^{2} x_{A}+\sqrt{P_{R}} \sqrt{(1-\beta) P_{B}} h_{A} h_{B} G x_{B}+ \\
& +\sqrt{P_{R}} G h_{A} n_{R}+v_{A}+n_{A}
\end{aligned}
$$

and

$$
\begin{aligned}
& y_{B}=\sqrt{P_{R}} h_{B} G\left(\sqrt{(1-\beta) P_{A}} h_{A} x_{A}+\sqrt{(1-\beta) P_{B}} h_{B} x_{B}+n_{R}\right)+ \\
& +v_{B}+n_{B} \\
& =\sqrt{P_{R}} \sqrt{(1-\beta) P_{B}} G\left|h_{B}\right|^{2} x_{B}+\sqrt{P_{R}} \sqrt{(1-\beta) P_{A}} h_{A} h_{B} G x_{A}+ \\
& +\sqrt{P_{R}} G h_{B} n_{R}+v_{B}+n_{B} .
\end{aligned}
$$

Next, the expression of SNDR at the source node $A$ is shown as below

$$
\gamma_{A 2}=\frac{P_{R}(1-\beta) P_{B} G^{2}\left|h_{A}\right|^{2}\left|h_{B}\right|^{2}}{P_{R} G^{2}\left|h_{A}\right|^{2} \sigma^{2}+P_{R}\left|h_{A}\right|^{2} \tau^{2}+\sigma^{2}} .
$$

Similarly to the derivation in the PSIR, we also obtain the closed-form expression of SNDR as follows

$$
\begin{aligned}
& \gamma_{A 2}^{*}= \\
& \frac{\left|h_{A}\right|^{2}\left|h_{B}\right|^{2}}{\frac{P_{A} \tau_{A}^{2}\left|h_{A}\right|^{4}}{(1-\beta) P_{B}}+\frac{\tau_{A}^{2}\left|h_{A}\right|^{2}\left|h_{B}\right|^{2}}{(1-\beta)}+\frac{\sigma^{2}\left(1+\tau_{A}^{2}\right)\left|h_{A}\right|^{2}}{(1-\beta) P_{B}}+\frac{\sigma^{2}}{\eta(1-\beta) P_{B} \beta}} .
\end{aligned}
$$

\section{Outage Probability and Throughput Analysis}

\subsection{Outage Probability}

We first derive the analytical expression for outage probability based on the Lemma 1 as given below.

Lemma 1: Let $m, n$ and $k$ be strictly positive constants, $\rho_{1}$ and $\rho_{2}$ be a non-negative random variables, $\lambda$ is the threshold of SNDR, $\Omega_{1}=\mathbb{E}\left\{\rho_{1}\right\}$ and $\Omega_{2}=\mathbb{E}\left\{\rho_{2}\right\}$ be average gain, the outage probability is computed by

$$
\begin{aligned}
& \operatorname{Pr}\left(\frac{\rho_{1} \rho_{2}}{m \rho_{1}^{2}+m \rho_{1} \rho_{2}+n \rho_{1}+k} \leq \lambda\right) \\
& =1-\frac{2}{\Omega_{1}} e^{-\frac{n \lambda}{\Omega_{2}(1-\lambda m)}} \times \sqrt{\frac{k \lambda}{\Omega_{2}(1-\lambda m)}\left(\frac{1}{\Omega_{2}} \frac{\lambda m}{1-\lambda m}+\frac{1}{\Omega_{1}}\right)^{-1}} \\
& \times K_{1}\left(2 \sqrt{\frac{k \lambda}{\Omega_{2}(1-\lambda m)}\left(\frac{1}{\Omega_{2}} \frac{\lambda m}{1-\lambda m}+\frac{1}{\Omega_{1}}\right)}\right)
\end{aligned}
$$

where $K_{1}($.$) stands for the first-order modified Bessel$ function of the second kind. For Proof: See Appendix.

Without loss of generality, we assume that $P_{A}=P_{B}=P$ and derive the outage probability of these proposed protocols in general form as below

$$
\gamma_{A}^{*}=\frac{\rho_{1} \rho_{2}}{m \rho_{1}^{2}+m \rho_{1} \rho_{2}+n \rho_{1}+k}
$$

where $\gamma_{A}^{*}$ denotes the upper bound of SNDR, $\rho_{j}=\left|h_{j}\right|^{2}$ and hence using the results of Lemma 1, we obtain the outage probability of received signal at each source node in the TWRN which is defined as $P_{\text {out }}^{A}=\operatorname{Pr}\left(\gamma_{A}^{*} \leq x\right)$, where $x$ is the threshold of SNDR, by changing the variables as below

$$
\begin{aligned}
& m=\frac{\tau_{R}^{2}}{1-\beta}, n=\frac{\sigma^{2}}{(1-\beta) P}, k=\frac{\sigma^{2}\left(1+\tau_{R}^{2}\right)}{P(1-\beta) \eta \beta}, \text { for PSIR. } \\
& m=\frac{\tau_{A}^{2}}{1-\beta}, n=\frac{\sigma^{2}\left(1+\tau_{A}^{2}\right)}{(1-\beta) P}, k=\frac{\sigma^{2}}{\eta(1-\beta) P \beta}, \text { for PSIS. }
\end{aligned}
$$

\subsection{Maximized Throughput Analysis}

In this paper, we focus on delay-limited transmission where the destination node needs to decode the received signal block by block and the code length is smaller than the transmission block time. In such transmission, the speed of transmitted signal is considered as fixed rate. Indeed, we can find the throughput based on the outage probability calculated in previous sections as 


$$
C^{\text {PSIR,PSIS }}=\frac{T / 2}{T}\left\{\left[1-P_{\text {out }}^{A}\left(R_{A}\right)\right] R_{A}+\left[1-P_{\text {out }}^{B}\left(R_{B}\right)\right] R_{B}\right\}
$$

where $C^{P S I R, P S I S}$ denotes the throughput for both PSIR and PSIS protocols, $P_{\text {out }}^{A}$ and $P^{B}{ }_{\text {out }}$ are the outage probabilities and $R_{\mathrm{A}}$ and $R_{\mathrm{B}}$ are the fixed rates at source nodes $A$ and $B$, respectively denoted as $R_{\mathrm{A}}=\log _{2}\left(1+\gamma_{\mathrm{A}}^{*}\right)$ and $R_{\mathrm{B}}=$ $\log _{2}\left(1+\gamma_{\mathrm{B}}^{*}\right)$. It is easy to verify that $C^{P S I R, P S I S}$ depends on $\beta, P, \tau_{R}, \tau_{A}, \sigma^{2}$ and $\lambda$. Consequently, for given parameters of $P, \tau_{R}, \tau_{A}, \sigma^{2}$ and $\lambda$ we can determine the maximum throughput by finding the optimal energy harvesting fractions of $\beta$.

Without loss of generality, we assume that $R_{A}=R_{B}=R$, $P_{\text {out }}^{A}\left(R_{A}\right)=P_{\text {out }}^{B}\left(R_{B}\right)=P_{\text {out }}$, and hence the throughput function can be expressed as below

$$
C^{P S I R, P S I S}=\left(1-P_{\text {out }}\right) R \text {. }
$$

\section{Numerical Results}

In this section, we discuss the performance of the analytical models through simulations, and investigate the impact of key system parameters on the throughout of the system. We set the source transmission rate applied to the high-rate wireless system as $4 \mathrm{bps} / \mathrm{Hz}$ (i.e., 4 bits/channel use), and hence the outage SNDR threshold is given by $\lambda=2^{4}-1$. Noise variances are kept fixed in all nodes, i.e., $\sigma_{A}=\sigma_{B}=\sigma_{R}=\sigma=1$ and the channel gains of the two source nodes $\Omega_{A}=\Omega_{B}=1$. Related to energy harvesting, we set the perfect energy conversion efficiency as $\eta=1$. Monte-Carlo simulations are used to validate the analytical expressions of outage probability and throughput. It is important to see that there is perfect agreement between Monte-Carlo simulations and analytical curves. In addition, these simulation results are obtained by evaluating expression over $10^{5}$ random iterations of the Rayleigh fading channels.

The simulation results in Fig. 1 and Fig. 2 further deal with the outage performance in the PSIR and PSIS protocols. We set the fraction of the received signal power for energy harvesting as $\beta=0.5$. These curves demonstrate that hardware impairments are critical impacts at high transmitted power. As can be seen, the hardware impairments are considered as critical parameters for evaluating the TWRN's performance, especially as these parameters increases, the performance of outage probability are decreased. Unfortunately, the outage probability curves at high level of hardware impairments approach the outage values.

We consider the case of variable fractions of harvested power for energy harvesting protocol. As shown in Fig. 3, the percentage of harvested power (i.e. $\beta$ ) directly influences the outage probability and then the optimal throughput. We set the transmitted power $P=20 \mathrm{~dB}$. Intuitively, the value of the throughput of the PSIR and the PSIS are roughly equivalent. Specifically, we consider the throughput performance in the PSIR and PSIS protocols, the throughput at destination increases as $\beta$ changes from 0 to the optimal value. This result is because smaller fraction of $\beta$ yields less harvested energy. Such less energy leads to decrease the outage performance and then obtaining larger throughput. In contrast, when $\beta$ increases over the optimal threshold, smaller value of $(1-\beta)$ in (11) and (16) results in smaller throughput at the destination node.

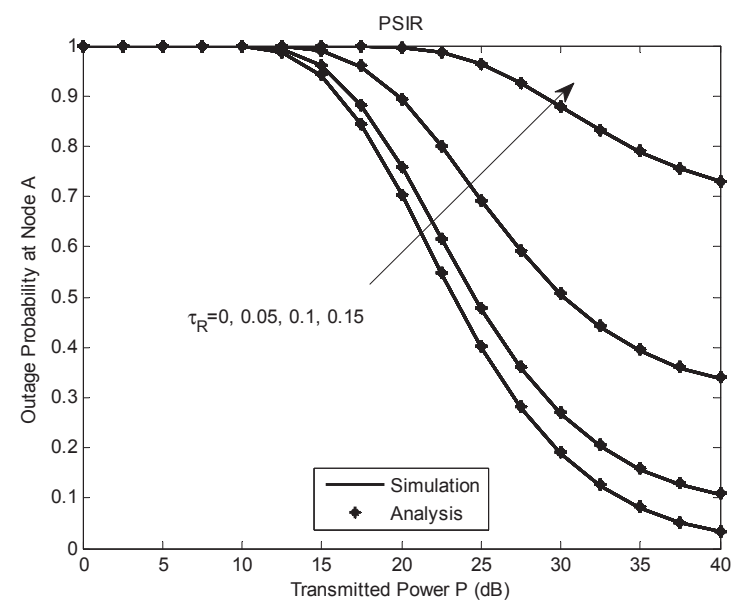

Fig. 1. Outage probability versus $P$ in the PSIR protocol.

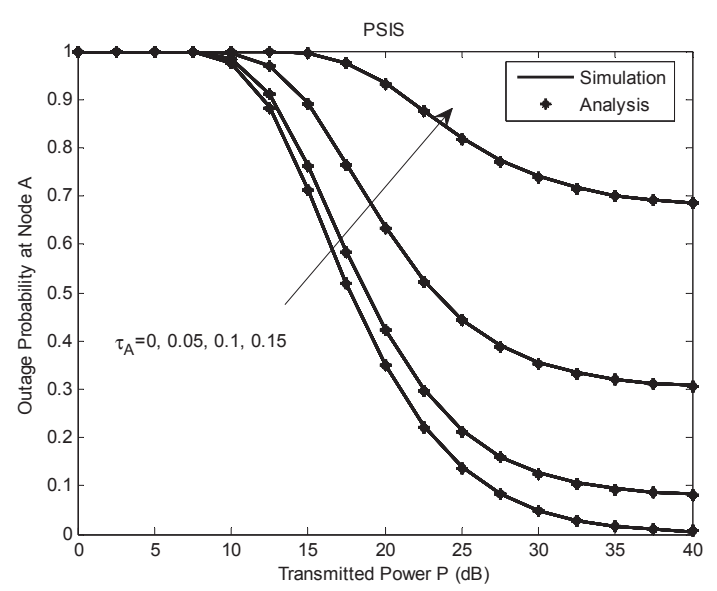

Fig. 2. Outage probability versus transmitted power $P$ in the PSIS protocol.

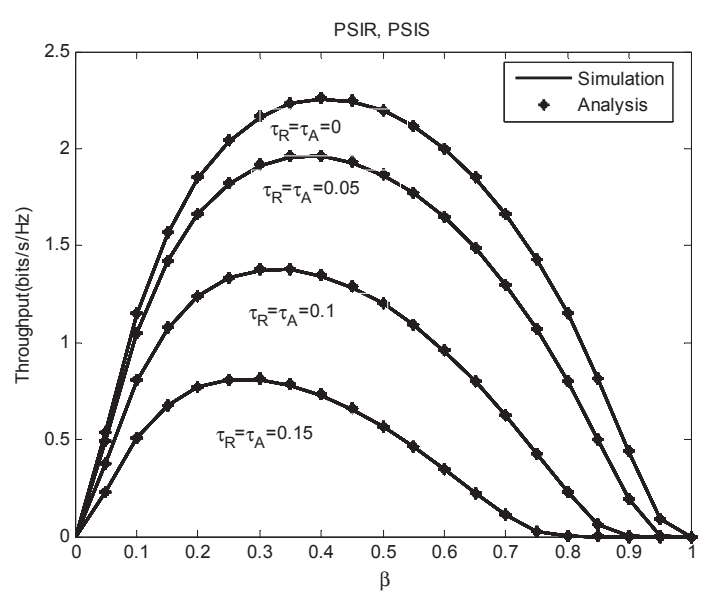

Fig. 3. Optimal throughput for the PSIR, PSIS protocol. 


\section{Conclusions}

This paper has presented how dramatic levels of hardware impairments affect the outage probability and the optimal throughput in the energy constraint TWRN where the wireless information and the power are transferred simultaneously. We have investigated several energy policies for harvesting energy at the relaying nodes which are often located far from the source nodes equipped with stable and powerful energy source. It has been shown in simulation results that analytical and simulated curves are matched completely. The important achievements are our derivations on optimal energy harvesting fractions for obtaining the maximized throughput. We derive the closedform and tractable expressions for the outage probability as the function of hardware impairments coefficients. Our results can provide helpful engineering guidelines to design transceiver circuits with acceptable hardware impairments thresholds in order to satisfy the outage probability and the maximized throughput requirements in real energy harvesting enabled cooperative wireless communications.

\section{Appendix}

\section{Proof of Lemma 1:}

It is worth mentioning that $\rho_{1}$ and $\rho_{2}$ are two independent random variables and we obtain the below formula using the law of total probability to condition on $\rho_{1}$ and $\rho_{2}$.

For instance, the probability to condition on $\rho_{1}$ can be written as

$$
\begin{aligned}
\mathrm{I} & \triangleq \operatorname{Pr}\left(\frac{\rho_{1} \rho_{2}}{m \rho_{1}^{2}+m \rho_{1} \rho_{2}+n \rho_{1}+k} \leq \lambda\right) \\
& =1-\int_{0}^{\infty} \operatorname{Pr}\left(\frac{\rho_{1} \rho_{2}}{m \rho_{1}^{2}+m \rho_{1} \rho_{2}+n \rho_{1}+k}>\lambda \mid \rho_{1}\right) \times f_{\rho_{1}}(x) d x .
\end{aligned}
$$

Next, we calculate conditional probability as the following expression if

$$
\begin{aligned}
& \operatorname{Pr}\left(\frac{\rho_{1} \rho_{2}}{m \rho_{1}^{2}+m \rho_{1} \rho_{2}+n \rho_{1}+k}>\lambda \mid \rho_{1}\right) \\
& =\left\{\begin{array}{cl}
1-F_{\rho_{2}}\left(\frac{\lambda\left(m \rho_{1}^{2}+n \rho_{1}+k\right)}{\rho_{1}(1-\lambda m)}\right), & \text { if } \lambda<\frac{1}{m} \\
0, & \text { if } \lambda \geq \frac{1}{m}
\end{array}\right.
\end{aligned}
$$

Substituting (A.2) into (A.1) and replacing (2), (3) into (A.1), new expression can be given by

$$
\begin{aligned}
I= & 1-\int_{0}^{\infty} \exp \left(-\frac{1}{\Omega_{2}} \frac{\lambda\left(m x^{2}+n x+k\right)}{x(1-\lambda m)}\right) \frac{1}{\Omega_{1}} \exp \left(-\frac{x}{\Omega_{1}}\right) d x \\
& =1-\frac{1}{\Omega_{1}} \exp \left(-\frac{n \lambda}{\Omega_{2}(1-\lambda m)}\right) \\
& \int_{0}^{\infty} \exp \left(-\left(\frac{\lambda m}{\Omega_{2}(1-\lambda m)}+\frac{1}{\Omega_{1}}\right) x-\frac{\lambda k}{\Omega_{2}(1-\lambda m)} \frac{1}{x}\right) d x .
\end{aligned}
$$

Finally, applying the following expression (3.324.1) in [22] $\int_{0}^{\infty} \exp \left(-\frac{\varphi}{4 x}-\varpi x\right) d x=\sqrt{\frac{\varphi}{\varpi}} K_{1}(\varphi \varpi)$, we obtain (17). This completes the proof of Lemma 1.

\section{References}

[1] PARK, J., CLERCKX, B. Joint wireless information and energy transfer in a two-user MIMO interference channel. IEEE Transactions on Communication, 2013, vol. 12, no.8, p. 4210-4221. DOI: $10.1109 /$ twc.2013.071913.130084.

[2] DOHLER, M., LI Y. Cooperative Communications: Hardware, Channel and PHY. John Wiley \& Sons, 2010.

[3] AHMED, I., IKHLEF, A., SCHOBER, R., MALLIK, R. K. Joint power allocation and relay selection in energy harvesting $\mathrm{AF}$ relay systems. IEEE Wireless Communication Letters, 2013, vol. 2, no. 2, p. 239-242. DOI: 10.1109/WCL.2013.012513.130007

[4] YIN, S., ZHANG, E., QU, Z., YIN, L., LI, S. Optimal cooperation strategy in cognitive radio systems with energy harvesting. IEEE Transactions on Communication, 2014, vol. 13, no. 9, p. 4693-4707. DOI: 10.1109/twc.2014.2322972

[5] WANG, Z., CHEN, Z., LUO, L., HU, Z., XIA, B., LIU, H. Outage analysis of cognitive relay networks with energy harvesting and information transfer. In Proc. of International Conference on Communication (ICC). Sydney (Australia), 2014, p. 4348-4352. DOI: 10.1109/icc.2014.6884004

[6] GU, Y., AISSA, S. Interference aided energy harvesting in Decode-and-Forward relaying systems. In Proc. of the IEEE International Conference on Communications (ICC). Sydney (Australia), 2014, p. 5378-5382. DOI: 10.1109/icc.2014.6884176

[7] ZHONG, C., SURAWEERA, H. A., ZHENG, G., KRIKIDIS, I., ZHANG, Z. Wireless information and power transfer with full duplex relaying. IEEE Transactions on Communication, 2014, vol. 62 , p. 3447-3461.

[8] DO, D. T. Time power switching based relaying protocol in energy harvesting mobile node: optimal throughput analysis. Mobile Information System, 2015, vol. 2015. DOI: $10.1155 / 2015 / 769286$

[9] TutuncuOGlu, K., VARAN, B., Yener, A. Optimum transmission policies for energy harvesting two-way relay. In Proc. of IEEE International Conference on Communications (ICC). Budapest (Hungary), 2013, p. 586-590. DOI: 10.1109/iccw.2013.6649301 
[10] NASIR, A., ZHOU, X., DURRANI, S., KENNEDY, R. A. Relaying protocols for wireless energy harvesting and information processing, IEEE Transactions on Wireless Communications, 2013, vol. 12, no. 7, p. 3622-3636. DOI: TWC.2013.062413.122042

[11] LUO, S., ZHANG, R., LIM, T. J. Optimal save-then-transmit protocol for energy harvesting wireless transmitters. IEEE Transactions on Wireless Communications, 2013, vol. 12, no. 3, p. 1196-1217. DOI: 10.1109/TWC.2013.012413.120488

[12] ZHOU, X., ZHANG, R., HO, C. K. Wireless information and power transfer: architecture design and rate-energy tradeoff. IEEE Transactions on Communications, 2013, vol. 61, no. 11, p. 4754-4767. DOI: 10.1109/TCOMM.2013.13.120855

[13] AL-QAHTANI, F. S., YANG, J., RADAYDEH, R. M., ALNUWEIRI, H. On the capacity of two-hop AF relaying in the presence of interference under Nakagami-m fading. IEEE Communication Letters, 2013, vol. 17, no. 1, p. 19-22. DOI: 10.1109/LCOMM.2012.111612.121151

[14] HWANG, K.-S., JU, M., ALOUINI, M.-S. On the outage performance of two-way Amplify-and-Forward relaying with outdated CSI over multiple relay network. In Proc. of IEEE 24th International Symposium on Personal, Indoor and Mobile Radio Comm. London (UK), 2013, p. 1-5. DOI: 10.1109/pimrc.2013.6666318

[15] IKKI, S. S., AISSA, S. Performance analysis of two-way amplify-and-forward relaying in the presence of co-channel interferences. IEEE Transactions on Communication, 2012, vol. 60, no. 4, p. 933-939. DOI: 10.1109/TCOMM.2012.013112.110188

[16] LOUIE, R., LI, Y., SURAWEERA, H. A., VUCETIC, B. Performance analysis of beamforming in two-hop amplify and forward relay networks with antenna correlation. IEEE Transactions on Wireless Communications, 2009, vol. 8, no. 6, p. 3131-3142. DOI: 10.1109/TWC.2009.080807

[17] NASIR, A. A., ZHOU, X., DURRANI, S., KENNEDY, R. A. Throughput and ergodic capacity of wireless energy harvesting based DF relaying network. In Proc. of IEEE International Conference on Communications (ICC). Sydney (Australia), 2014, p. 1-6. DOI: 10.1109/icc.2014.6883957

[18] LI, J., MATTHAIOU, M., SVENSSON, T. I/Q imbalance in two-way AF relaying. IEEE Transactions on Communication,
2014, vol. 62, no. 7, p. 2271-2285. DOI: 10.1109/TCOMM.2014.2325036

[19] MEDARD, M. The effect upon channel capacity in wireless communications of perfect and imperfect knowledge of the channel. IEEE Transaction on Communication, 2000, vol. 46, no. 3, p. 933-946. DOI: $10.1109 / 18.841172$

[20] LIU, Y., WANG, L., ELKASHLAN, M., DUONG, T. Q., NALLANATHAN, A. Two-way relaying networks with wireless power transfer: policies design and throughput analysis. In Proc. of IEEE Global Communications Conference (GLOBECOM'14). Austin (USA, TX), 2014, p. 4030-4035. DOI: 10.1109/glocom.2014.7037438

[21] POPOVSKI, P., YOMO, H. Physical network coding in two-way wireless relay channels. In Proc. of International Conference on Communication (ICC). Glasgow (UK), June 2007, p. 707-712. DOI: $10.1109 /$ icc.2007.121

[22] GRADSHTEYN, I. S., RYZHIK, I. M. Table of Integrals, Series, and Products. New York, NY, USA: Academic, 2000.

\section{About the Author ...}

Dinh-Thuan DO received the B.S. degree, M. Eng. degree, and Ph.D. degree from Vietnam National University (VNU-HCMC) in 2003, 2007, and 2013 respectively, all in Communications Engineering. He was a visiting Ph.D. student with Communications Engineering Institute, National Tsing Hua University, Taiwan from 2009 to 2010. Prior to joining Duy Tan University, he was senior engineer at the VinaPhone Mobile Network from 2003 to 2009. Since 2014, he is serving as an assistant professor in Wireless Communications Lab, Duy Tan University. His research interest includes signal processing in wireless communications network, cooperative communications, full-duplex transmission and energy harvesting. Dr. Thuan has published over 35 technical papers in international journals and conferences and served as an active TPC member of several international conferences. 